03

\title{
Малоугловое рассеяние и поляризация излучения вытянутой полимерной пленкой с каплями нематического жидкого кристалла, имеющими монодоменную структуру
}

\author{
(C) В.А. Лойко ${ }^{1}$, А.В. Конколович ${ }^{1}$, А.А. Мискевич ${ }^{1}$, М.Н. Крахалев ${ }^{2,3}$, \\ О.О. Прищепа ${ }^{2,3}$, В.Я. Зырянов ${ }^{2}$ \\ ${ }^{1}$ Институт фризики им. Б.И. Степанова НАН Беларуси, \\ 220072 Минск, Беларусь \\ ${ }^{2}$ Институт физики им. Л.В. Киренского ФИЦ КНЦ Сибирского отделения РАН, \\ 660036 Красноярск, Россия \\ ${ }^{3}$ Институт инженерной фризики и радиоэлектроники, Сибирский фредеральный университет, \\ 660041 Красноярск, Россия \\ e-mail: loiko@ifanbel.bas-net.by
}

Поступила в редакцию 01.11.2019 г.

В окончательной редакции 01.11.2019 г.

Принята к публикации 21.11.2019 г.

Исследованы коэффициент направленного пропускания вытянутой капсулированной полимером жидкокристаллической пленки с бездефектной монодоменной структурой капель жидкого кристалла, формируемой при растяжении пленки, допированной сурфактантами, и степень поляризации прямопрошедшего излучения в видимом и ближнем инфракрасном диапазонах спектра. Приведены результаты для нематических жидких кристаллов 5СВ, Е7 и Е44. Исследованы зависимости коэффициента пропускания и поляризующей способности пленки от угла поля зрения фотоприемника. В приближениях Фолди-Тверского и аномальной дифракции получены соотношения, позволяющие определять параметры пленок, при которых их коэффициент пропускания и поляризуюшая способность одновременно достигают значений, близких к предельным (0.5 и \pm 1.0 соответственно).

Ключевые слова: рассеяние света, поляризация излучения, жидкий кристалл

DOI: $10.21883 /$ OS.2020.03.49062.296-19

\section{Введение}

Использование капсулированных полимером жидкокристаллических (КПЖК) пленок для поляризации светового излучения позволяет существенно увеличить предельную мощность падающего светового потока [1]. Они позволяют поляризовать излучение с плотностью мощности падающего потока до $2 \mathrm{~kW} / \mathrm{cm}^{2}$ [2,3] и выше, когда обычные пленочные поляризаторы (поляроиды) [4] с анизотропией поглощения разрушаются вследствие нагрева. КПЖК пленки, состоящие из вытянутых ориентированных капель жидкого кристалла (ЖК) в полимерной матрице [1-3,5-10], обладают анизотропией светорассеяния и эффективно поляризуют излучение во всей области прозрачности используемых компонентов (видимая и ближняя ИК области), в то время как поляроиды - только в дихроичной полосе собственного или примесного поглощения. Для таких пленок появляется дополнительная возможность управления и модуляции оптического отклика воздействием электрического или магнитного поля. Они особенно перспективны для применения в лазерных и проекционных устройствах.

Недавно реализован новый метод управления оптическим откликом КПЖК пленок в светорассеивающей моде, который основан на локальном эффекте
Фредерикса [11-14]. Суть эффекта заключается в том, что структура поля директора капель нематического ЖК изменяется благодаря неоднородному межфазному поверхностному сцеплению на границе капля-полимер. Неоднородность сцепления создается путем использования поверхностно активных веществ (сурфактантов). Такой способ управления внутренней структурой капель позволяет сформировать в них близкую к однородной (монодоменной) ориентацию локальных оптических осей при механическом растяжении пленки. Это приводит к существенному увеличению эффективности поляризации света [15-17] в сравнении со способами, основанными на однородном межфазном поверхностном сцеплении.

Оптико-механическая модель описания коэффициента когерентного (направленного) пропускания и поляризующей способности КПЖК пленки с вытянутыми каплями жидкого кристалла разработана в [17]. Она основана на приближениях Фолди-Тверского и аномальной дифракции. Модель описывает оптический отклик вытянутой пленки в зависимости от ее толщины, показателя преломления полимера, размеров, параметров анизометрии ЖК капель, их концентрации, внутренней структуры, полидисперсности и ориентации оптических осей. Однако она рассматривает только коэффициент на- 
правленного пропускания пленки и степень поляризации прямопрошедшего излучения.

В настоящей работе разработана модель, которая учитывает не только прямопрошедшее, но и рассеянное излучение. Она обобщает результаты, описанные в [17]. Модель позволяет анализировать (i) угловое распределение света, рассеянного в переднюю полусферу, (ii) пропускание и поляризующую способность вытянутых КПЖК пленок с учетом угла поля зрения приемника.

Проведен численный анализ спектральных зависимостей коэффициента когерентного пропускания и поляризующей способности вытянутой непоглощающей КПЖК пленки с монодоменной структурой капель при ионносурфактантной модификации межфазного поверхностного сцепления в видимом и ближнем инфракрасном диапазонах спектра. Исследовано влияние показателя преломления полимерной матрицы на спектральные зависимости пропускания и поляризующей способности КПЖК пленки, содержащей капли нематических жидких кристаллов 5СВ, Е7, Е44, и влияние угла приема рассеянного света на пропускание и поляризующую способность.

\section{Основные соотношения}

\section{Коэффициенты когерентного пропускания и поляризующая способность вытянутой пленки}

Схематическое изображение структуры КПЖК слоя при растяжении вдоль оси у представлено на рис. 1. Плоскопараллельный КПЖК слой при однонаправленном механическом растяжении вдоль оси $y$ освещается по нормали (вдоль оси $x$ ) неполяризованным излучением. Плоскость $y z$ совпадает с передней поверхностью слоя; $\mathbf{N}_{j}$ - оптическая ось (директор) $j$-й капли; $\varphi-$ угол ориентации оптической оси капли относительно оси $y$; $a, b, c$ - полуоси вытянутых эллипсоидальных капель; $l$ - толщина растянутого слоя; $l_{y}, l_{z}$ - его линейные размеры вдоль осей $y, z$ лабораторной системы координат $x y z$.

Определим коэффициент когерентного (направленного) пропускания $T_{c}^{\mathrm{np}}$ и поляризующую способность (степень поляризации прямопрошедшего света) $P_{c}$ слоя следующим образом:

$$
\begin{gathered}
T_{c}^{\mathrm{np}}=\frac{T_{\|}^{c}+T_{\perp}^{c}}{2}, \\
P_{c}=\frac{T_{\perp}^{c}-T_{\|}^{c}}{T_{\|}^{c}+T_{\perp}^{c}},
\end{gathered}
$$

где $T_{\|}^{c}$ и $T_{\perp}^{c}-$ коэффициенты пропускания слоя, определяемые в параллельных поляризаторе и анализаторе при их расположении соответственно вдоль и ортогонально оси растяжения $y$.

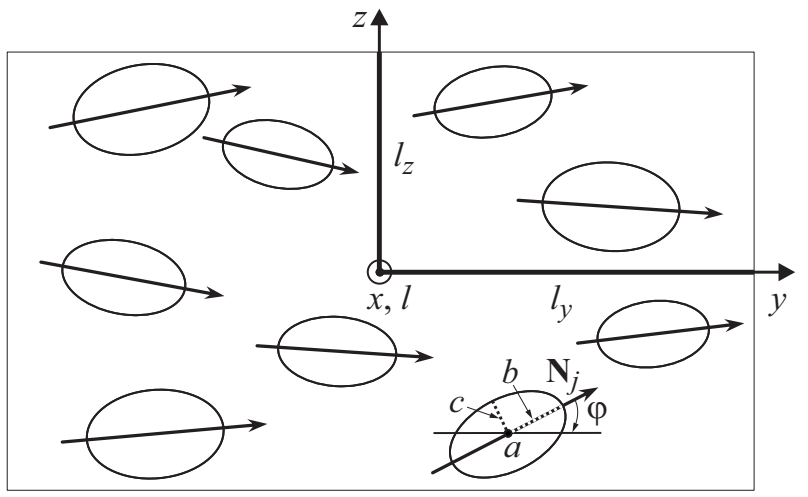

Рис. 1. Схематическое изображение структуры КПЖК слоя при растяжении вдоль оси $y . x y z$ - лабораторная система координат; плоскость $y z$ совпадает с передней поверхностью слоя; $\varphi-$ угол ориентации оптической оси $\mathbf{N}_{j}$ отдельной капли; $l$ и $l_{y}, l_{z}$ - толщина растянутого слоя и его линейные размеры вдоль осей $y$ и $z ; a, b, c-$ полуоси вытянутых капель в форме эллипсоидов. Слой освещается по нормали (вдоль оси $x$ ) неполяризованным излучением.

Используя приближение Фолди-Тверского [17,18], запишем:

$$
\begin{gathered}
T_{\|, \perp}^{c}=\exp \left(-\gamma_{2,1} l\right), \\
\gamma_{2}=\frac{4 \pi}{k^{2}} N_{v}\left\langle\operatorname{Re} S_{e}^{0} \cos ^{2} \varphi+\operatorname{Re} S_{o}^{0} \sin ^{2} \varphi\right\rangle_{a, b, c, \mathbf{N}_{j}}, \\
\gamma_{1}=\frac{4 \pi}{k^{2}} N_{v}\left\langle\operatorname{Re} S_{e}^{0} \sin ^{2} \varphi+\operatorname{Re} S_{o}^{0} \cos ^{2} \varphi\right\rangle_{a, b, c, \mathbf{N}_{j}},
\end{gathered}
$$

где $\gamma_{2}$ и $\gamma_{1}-$ показатели ослабления слоя для $y$ и $z$-поляризаций падающего света; $k=2 \pi n_{p} / \lambda, n_{p}-$ показатель преломления полимерной матрицы, $\lambda$ длина волны падающего света, $N_{v}$ - число капель ЖК в единице объема, $S_{e, o}^{0}$ - элементы амплитудной матрицы рассеяния отдельной капли [18-20] при нулевом угле рассеяния для необыкновенной (индекс $e$ ) и обыкновенной (индекс $o$ ) волн с поляризациями вдоль и поперек оптической оси $\mathbf{N}_{j}$. При одинаковой внутренней структуре капель угловые скобки в выражениях (4), (5) означают усреднение по размерам капель $a, b, c$ и ориентации их оптических осей $\mathbf{N}_{j}$.

Предположим, что до растяжения слой состоит из полидисперсных капель в форме сфероидов (или сфер) с полуосями $a_{0}$ и $c_{0}$. Полуоси $a_{0}$ ориентированы вдоль оси $x$; полуоси $c_{0}$ ориентированы параллельно плоскости $y z$. Для сфероидов $a_{0}<c_{0}$ (для сфер $a_{0}=c_{0}$ ). Считаем, что параметры анизометрии (отношения осей) одинаковы для всех капель в исходном состоянии слоя и изменяются одинаково в процессе растяжения. Тогда, используя приближение аномальной дифракции для элементов амплитудной матрицы рассеяния $S_{e, o}^{0}$ и теорему о среднем [19,21], запишем выражения для показателей ослабления $\gamma_{2,1}$ :

$$
\gamma_{2}=\frac{3 c_{v}}{4 a_{\mathrm{ef}}}\left\{Q_{e}\left(a_{\mathrm{ef}}\right) \frac{1+S_{2 f}}{2}+Q_{o}\left(a_{\mathrm{ef}}\right) \frac{1-S_{2 f}}{2}\right\},
$$




$$
\begin{gathered}
\gamma_{1}=\frac{3 c_{v}}{4 a_{\mathrm{ef}}}\left\{Q_{e}\left(a_{\mathrm{ef}}\right) \frac{1-S_{2 f}}{2}+Q_{o}\left(a_{\mathrm{ef}}\right) \frac{1+S_{2 f}}{2}\right\}, \\
Q_{e, o}\left(a_{\mathrm{ef}}\right)=2 \operatorname{Re} \int_{\sigma=\pi b c}\left(1-T_{2,1}\left(a_{\mathrm{ef}}\right) d \sigma\right), \\
S_{2 f}=2\left\langle\cos ^{2} \varphi\right\rangle_{\varphi}-1, \\
a_{\mathrm{ef}}=\left\langle a^{3}\right\rangle /\left\langle a^{2}\right\rangle .
\end{gathered}
$$

В формуле (9) угловые скобки означают усреднение по углу $j$ ориентации оптических осей $\mathbf{N}_{j}$ (рис. 1), в формуле (10) - по размеру а полуосей капель. $c_{v}$ - объемный фактор заполнения слоя (отношение объема всех капель к объему слоя, в котором они распределены); $a_{\mathrm{ef}}-$ эффективное значение длины полуоси $a ; Q_{e, o}\left(a_{\mathrm{ef}}\right)-$ факторы эффективности ослабления отдельной капли для необыкновенной и обыкновенной волн; $T_{2,1}$ - диагональные элементы матрицы Джонса эквивалентного амплитудно-фазового экрана; $S_{2 f}-$ двумерный (2D) параметр порядка КПЖК слоя.

При растяжении пленки ее толщина $l$, размеры капель $a, b, c$ и параметры анизометрии $\varepsilon_{y}=b / a$, $\varepsilon_{z}=c / a$ изменяются следующим образом [22]:

$$
\begin{gathered}
l=l_{0} p^{-B}, \\
a=a_{0} p^{-B}, \quad b=c_{0} p, \quad c=c_{0} p^{-A}, \\
\varepsilon_{y}=b / a=\left(c_{0} / a_{0}\right)^{1+B}, \quad \varepsilon_{z}=c / a=\left(c_{0} / a_{0}\right)^{B-A},
\end{gathered}
$$

где $p=l_{y} / l_{y}^{0}$ - коэффициент растяжения, равный отношению длин $l_{y}$ и $l_{y}^{0}$ рассматриваемого участка слоя в деформированном $(p \neq 1)$ и исходном $(p=1)$ состояниях, показатели степени $A$ и $B$ зависят от механических свойств полимерной матрицы $(A+B=1)$.

Для вытянутых эллипсоидальных капель с монодоменной внутренней конфигурацией ЖК можно записать аналитические соотношения [23], описывающие факторы эффективности ослабления $Q_{e, o}$ в зависимости от размеpa (эффективного размера полуоси $a$ ) $a_{\mathrm{ef}}$ :

$$
Q_{e, o}\left(a_{\mathrm{ef}}\right)=4 \operatorname{Re} K_{e, o}\left(a_{\mathrm{ef}}\right),
$$

где $K-$ функция Хюлста [19],

$$
\begin{gathered}
K_{e, o}\left(a_{\mathrm{ef}}\right)=\frac{1}{2}+\frac{\exp \left(-\Delta_{e, o}\left(a_{\mathrm{ef}}\right)\right)}{i \Delta_{e, o}\left(a_{\mathrm{ef}}\right)}+\frac{\exp \left(-i \Delta_{e, o}\left(a_{\mathrm{ef}}\right)\right)-1}{\left(i \Delta_{e, o}\left(a_{\mathrm{ef}}\right)\right)^{2}} \\
\Delta_{e, o}\left(a_{\mathrm{ef}}\right)=2 k a_{\mathrm{ef}}\left(\frac{n_{\|, \perp}}{n_{p}}-1\right) .
\end{gathered}
$$

При анализе пропускания и поляризации мы полагали, что в рассмотренном диапазоне длин волн показатель преломления полимера намного слабее зависит от длины волны, чем показатели преломления жидкого кристалла, и считали его постоянным. Учет зависимости показателей преломления ЖК $n_{\|}$(необыкновенного)
Значения коэффициентов $A_{\|, \perp}, B_{\|, \perp}, C_{\|, \perp}$, для ЖК 5 СВ, Е7, E44 [24]

\begin{tabular}{c|c|c|c|c|c|c}
\hline LC & $A_{\|}$ & $B_{\|}$ & $C_{\|}$ & $A_{\perp}$ & $B_{\perp}$ & $C_{\perp}$ \\
\hline 5CB & 1.6708 & 0.0081 & 0.0024 & 1.5139 & 0.0052 & 0.0008 \\
E7 & 1.6933 & 0.0078 & 0.0028 & 1.4990 & 0.0072 & 0.0003 \\
E44 & 1.7282 & 0.0121 & 0.0027 & 1.5006 & 0.0091 & 0.0001
\end{tabular}

и $n_{\perp}$ (обыкновенного) от длины волны $\lambda$ проведен нами с использованием формулы Коши:

$$
n_{\|, \perp}=A_{\|, \perp}+\frac{B_{\|, \perp}}{\lambda^{2}}+\frac{C_{\|, \perp}}{\lambda^{4}} .
$$

Значения коэффициентов $A_{\|, \perp}, B_{\|, \perp}, C_{\|, \perp}$ для исследованных в данной работе нематических ЖК приведены в таблице.

\section{Интенсивность и степень поляризации некогерентно рассеянного света}

Интенсивность $I_{\text {np }}^{\text {inc }}$, степень поляризации $P_{\text {inc }}$, параллельная $I_{\|}^{\text {inc }}$ и ортогональная $I_{\perp}^{\text {inc }}$ оси растяжения компоненты интенсивности некогерентно (диффузно) рассеянного излучения при освещении неполяризованным излучением определяются следующим образом:

$$
\begin{gathered}
I_{\mathrm{np}}^{\mathrm{inc}}=\frac{1}{2}\left(I_{\|}^{\mathrm{inc}}+I_{\perp}^{\mathrm{inc}}\right), \\
P_{\mathrm{inc}}=\frac{I_{\perp}^{\mathrm{inc}}-I_{\|}^{\mathrm{inc}}}{I_{\|}^{\mathrm{inc}}+I_{\perp}^{\mathrm{inc}}}, \\
I_{\|, \perp}^{\mathrm{inc}}=\left.\left(I_{v v}^{\mathrm{inc}}+I_{v h}^{\mathrm{inc}}\right)\right|_{\alpha=0, \pi / 2} .
\end{gathered}
$$

Здесь $I_{v v}^{\text {inc }}$ и $I_{v h}^{\text {inc }}-v v$ - и $v h$-компоненты интенсивности излучения, рассеянного пленкой, освещаемой линейно поляризованной волной $(v v-$ компонента поляризации, параллельная плоскости поляризации падающей волны, $v h$ - компонента поляризации, ортогональная плоскости поляризации падающей волны); $\alpha-$ угол поляризации (угол между плоскостью поляризации падающей волны и осью растяжения $y$ ).

Если фактор заполнения $c_{v}$ слоя мал $\left(c_{v}<0.2\right)$, то можно использовать приближение однократного рассеяния [19]. В рамках этого приближения

$I_{v v, v h}^{\mathrm{inc}}\left(\theta_{s}, \varphi_{s}\right)=C \frac{3}{4} \frac{l\left\langle a^{2}\right\rangle}{\varepsilon_{y} \varepsilon_{z}\left\langle a^{3}\right\rangle} \frac{c_{v}}{k^{2}\langle\sigma\rangle}\left\langle\left|f_{v v, v h}\left(\theta_{s}, \varphi_{s}\right)\right|^{2}\right\rangle_{a, \mathbf{N}_{j}}$,

где $\theta_{s}$ и $\varphi_{s}$ - полярный и азимутальный углы рассеяния; $C=\left|E_{i}^{2}\right| A / R^{2}, E_{i}$ - амплитуда падающей волны, $A-$ площадь освещаемого участка слоя; $R-$ расстояние от слоя до точки наблюдения; $\sigma=\pi b c-$ сечение капель в плоскости слоя $y z ; f_{v v}\left(\theta_{s}, \varphi_{s}\right)$ и $f_{v h}\left(\theta_{s}, \varphi_{s}\right)-$ $v v$ - и $v h$-компоненты векторной амплитудной функции 

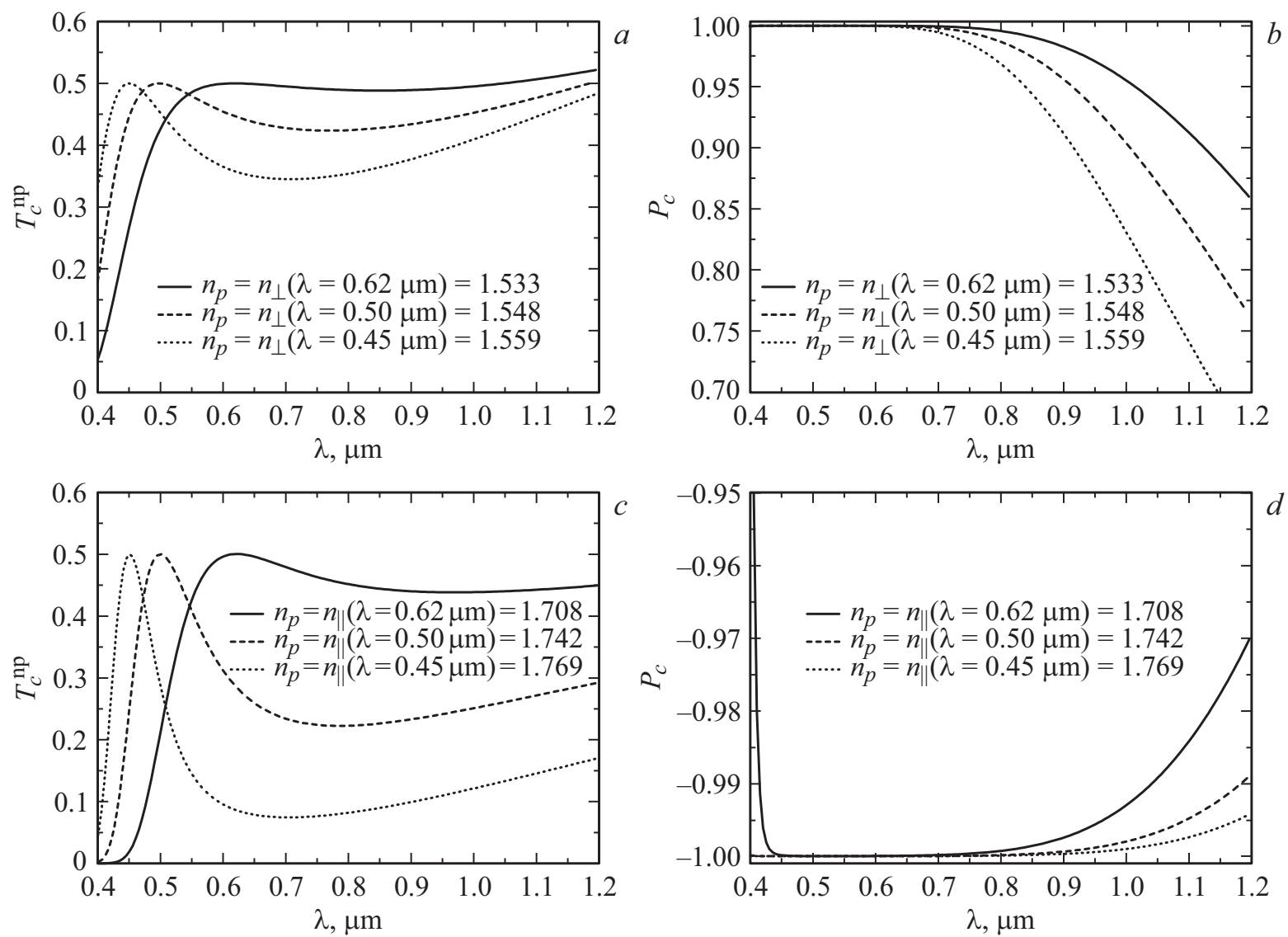

Рис. 2. Спектральные зависимости $T_{c}^{\mathrm{np}}(\lambda)(a, c)$ и $P_{c}(\lambda)(b, d)$. Показатель преломления полимера $n_{p}=n_{\perp}(a, b)$ и $n_{p}=n_{\|}(c, d)$ на длинах волн $\lambda=0.62,0.5,0.45 \mu \mathrm{m}$. ЖК 5СВ. $p=2$. Монодисперсные ориентированные капли $\left(S_{2 f}=1\right)$ с монодоменной структурой. LC 5CB, $l=32 \mu \mathrm{m}, c_{v}=0.143, a=0.7 \mu \mathrm{m}, \varepsilon_{y}=2.83, \varepsilon_{z}=1.0$.

рассеяния отдельной ЖК капли (определяемые в параллельных и скрещенных поляризаторе и анализаторе соответственно); угловые скобки $\langle\ldots\rangle_{a, \mathbf{N}_{j}}$ означают усреднение по размеру $а$ и ориентации оптических осей капель $\mathbf{N}_{j}$.

\section{Некогерентное пропускание и поляризующая способность в зависимости от апертурного угла приема}

Используя угловое распределение интенсивности рассеянного света (21), запишем формулы, описывающие зависимость коэффициента некогерентного пропускания $T_{\mathrm{np}}^{\mathrm{inc}}\left(\theta_{f o v}\right)$ и поляризующей способности $P_{\mathrm{inc}}\left(\theta_{f o v}\right)$ КПЖК пленки от апертурного угла приема $\theta_{f o v}$ :

$$
\begin{gathered}
T_{\mathrm{np}}^{\mathrm{inc}}\left(\theta_{f o v}\right)=\frac{1}{2}\left(T_{\|}^{\mathrm{inc}}\left(\theta_{\text {fov }}\right)+T_{\perp}^{\mathrm{inc}}\left(\theta_{f o v}\right)\right), \\
P_{\mathrm{inc}}\left(\theta_{f o v}\right)=\frac{T_{\perp}^{\mathrm{inc}}\left(\theta_{f o v}\right)-T_{\|}^{\mathrm{inc}}\left(\theta_{f o v}\right)}{T_{\|}^{\mathrm{inc}}\left(\theta_{f o v}\right)+T_{\perp}^{\mathrm{inc}}\left(\theta_{f o v}\right)},
\end{gathered}
$$

$$
\begin{aligned}
T_{\|, \perp}^{\mathrm{inc}}\left(\theta_{f o v}\right)= & \frac{1}{C_{\mathrm{np}}} \int_{0}^{2 \pi} d \varphi_{s} \int_{0}^{\theta_{f o v} / 2}\left(I_{v v}^{\mathrm{inc}}\left(\theta_{s}, \varphi_{s}\right)\right. \\
& \left.+I_{v h}^{\mathrm{inc}}\left(\theta_{s}, \varphi_{s}\right)\right)\left.\right|_{\alpha=0, \pi / 2} \sin \theta_{s} d \theta_{s} .
\end{aligned}
$$

Здесь $T_{\|}^{\text {inc }}\left(\theta_{f o v}\right)$ и $T_{\perp}^{\text {inc }}\left(\theta_{f o v}\right)-$ параллельная и ортогональная компоненты коэффициента некогерентного пропускания слоя (поляризатор и анализатор параллельны и расположены вдоль $(\alpha=0)$ и ортогонально $(\alpha=\pi / 2)$ оси $y$ лабораторной системы координат соответственно),

$$
\begin{aligned}
C_{\mathrm{np}} & =C \frac{3}{4} \frac{c_{v} l\left\langle a^{2}\right\rangle}{\varepsilon_{y} \varepsilon_{z}\left\langle a^{3}\right\rangle}\left(\left\langle Q_{\|}\right\rangle+\left\langle Q_{\perp}\right\rangle\right), \\
\left\langle Q_{\|, \perp}\right\rangle= & \left(\left\langle Q_{e}\right\rangle \frac{1 \pm S_{2 f}}{2}+\left\langle Q_{o}\right\rangle \frac{1 \mp S_{2 f}}{2}\right), \\
& \left\langle Q_{\|}\right\rangle+\left\langle Q_{\perp}\right\rangle \equiv\left\langle Q_{e}\right\rangle+\left\langle Q_{o}\right\rangle,
\end{aligned}
$$

где $\left\langle Q_{\|}\right\rangle$и $\left\langle Q_{\perp}\right\rangle$ - средние значения факторов эффективности ослабления ЖК капель для поляризаций падающего света, параллельной и ортогональной оси растяжения; $\left\langle Q_{e}\right\rangle$ и $\left\langle Q_{o}\right\rangle$ определяются по формуле (14). 

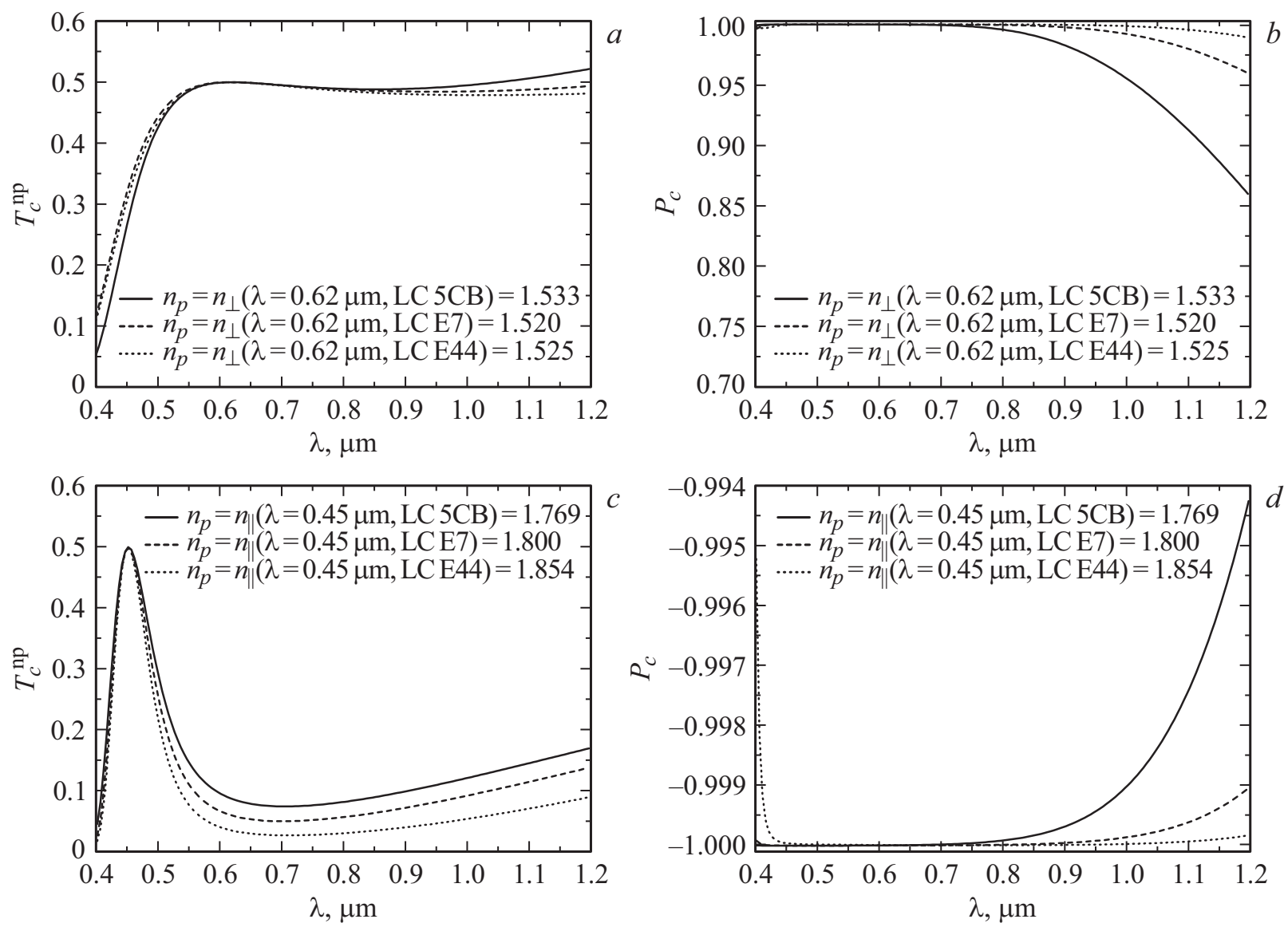

Рис. 3. Спектральные зависимости $T_{c}^{\mathrm{np}}(\lambda)(a, c)$ и $P_{c}(\lambda)(b, d)$. Показатель преломления полимера $n_{p}=n_{\perp}(a, b)$ и $n_{p}=n_{\|}(c, d)$ на длинах волн $\lambda=0.62 \mu \mathrm{m}(a, b)$ и $\lambda=0.45 \mu \mathrm{m}(c, d)$. ЖК 5СВ, Е7, Е44. $p=2$. Монодисперсные ориентированные капли $\left(S_{2 f}=1\right)$ с монодоменной структурой. $l=32 \mu \mathrm{m}, c_{v}=0.143, a=0.7 \mu \mathrm{m}, \varepsilon_{y}=2.83, \varepsilon_{z}=1.0$.

Для определения коэффициента пропускания $T_{\mathrm{np}}\left(\theta_{\text {fov }}\right)$ слоя и степени поляризации $P_{\text {fov }}$ света необходимо учесть когерентную $T_{c}^{\mathrm{np}}$ и некогерентную $T_{\mathrm{np}}^{\mathrm{inc}}\left(\theta_{f o v}\right)$ составляющие. Коэффициент пропускания и степень поляризации определяются следующим образом:

$$
\begin{gathered}
T_{\mathrm{np}}\left(\theta_{f o v}\right)=T_{\mathrm{np}}^{c}+T_{\mathrm{np}}^{\mathrm{inc}}\left(\theta_{f o v}\right)=T_{\|}\left(\theta_{\text {fov }}\right)+T_{\perp}\left(\theta_{f o v}\right), \\
P_{f o v}\left(\theta_{f o v}\right)=\frac{T_{\perp}\left(\theta_{\text {fov }}\right)-T_{\|}\left(\theta_{\text {fov }}\right)}{T_{\|}\left(\theta_{f o v}\right)+T_{\perp}\left(\theta_{f o v}\right)} \\
T_{\|, \perp}\left(\theta_{f o v}\right)=\frac{1}{2}\left(T_{\|, \perp}^{c}+T_{\|, \perp}^{\mathrm{inc}}\left(\theta_{f o v}\right)\right) .
\end{gathered}
$$

\section{Результаты расчетов}

В данном разделе приведены результаты анализа спектральных зависимостей коэффициента пропускания $T_{c}^{\mathrm{np}}$ и поляризуюшей спрособности $P$ КПЖК пленки при кратности растяжения $p=2.0$, соответствующей образованию монодоменных структур капель при ионносурфактантной модификации межфазного поверхностного сцепления [17].
Анализ записанных выше соотношений показывает, что основными параметрами, определяющими предельные поляризационные характеристики КПЖК пленки (пропускание $T_{c}^{\mathrm{np}}=0.5$ и степень поляризации $\left.P_{c}= \pm 1\right)$, являются: (i) показатель преломления полимерной матрицы $n_{p}$, который в образце должен быть равен обыкновенному показателю преломления жидкого кристалла $n_{\perp}$ (или необыковенному $n_{\|}$); (ii) поперечный (направлению растяжения пленки) размер ЖК капель $a$, который вместе с условием $n_{p}=n_{\perp}$ (или $n_{p}=n_{\|}$) определяет возможность одновременного достижения предельных значений для $T_{c}^{\mathrm{np}}$ и $P_{c}$.

Рис. 2 иллюстрирует зависимости $T_{c}^{\mathrm{np}}(\lambda)$ и $P_{c}(\lambda)$ для ЖК 5СВ при $a=0.7 \mu \mathrm{m}$, когда $n_{p}=n_{\perp}(\lambda)$ или $n_{p}=n_{\|}(\lambda)$ на длинах волн $\lambda=0.62,0.5,0.45 \mu \mathrm{m}$. Видно, что изменение показателя преломления полимера $n_{p}$ от $n_{p}=n_{\perp}$ до $n_{p}=n_{\|}$позволяет достигать высокого пропускания и эффективной поляризации света в широкой спектральной области. Выбранное значение поперечной полуоси капель $(a=0.7 \mu \mathrm{m})$ является оптимальным для достижения значений $T_{c}^{\mathrm{np}}$ и $P$, близких к предельным $\left(T_{c}^{\mathrm{np}} \approx 0.5, P_{c} \approx \pm 1.0\right)$ для пленки, параметры которой даны в [17]. 
На рис. 3 представлены зависимости $T_{c}^{\mathrm{np}}(\lambda)$ и $P_{c}(\lambda)$ для КПЖК пленки на основе разных ЖК: 5СВ, Е7, Е44. Видно, что увеличение оптической анизотропии ЖК, которая больше для ЖК Е44 в сравнении с Е7 и 5СВ, позволяет увеличить диапазон длин волн с предельной поляризующей способностью пленки для направленного света $P_{c} \approx \pm 1.0$ (рис. $3, b, d$ ).

Отметим, что положительные значения $P_{c}$ означают, что прямопрошедший свет линейно поляризован ортогонально направлению растяжения пленки; отрицательные значения $P_{c}$ означают, что свет поляризован параллельно направлению растяжения. Знак поляризации определяется тем, какая из компонент, ортогональная или параллельная оси растяжения, проходит пленку без рассеяния.

На рис. 4 и 5 представлены результаты расчета угловых распределений нормированной интенсивности рассеянного света

$$
I_{\mathrm{np}}^{\mathrm{norm}}\left(\theta_{s}, \varphi_{s}\right)=I_{\mathrm{np}}^{\mathrm{inc}}\left(\theta_{s}, \varphi_{s}\right) / I_{\mathrm{np}}^{\mathrm{inc}}\left(\theta_{s}=0, \varphi_{s}=0\right)
$$

и поляризующей способности $P_{\text {inc }}\left(\theta_{s}, \varphi_{s}\right)$ пленки при коэффициенте растяжения $p=2$.

Они получены для пленок $\mathrm{c}$ монодисперсными ориентированными каплями, у которых полуось $a=0.7 \mu \mathrm{m}$. Показатель преломления полимера $n_{p}=n_{\perp}(\lambda=0.62 \mu \mathrm{m})=1.533$. Необыкновенный показатель преломления ЖК $n_{\|}(\lambda=0.62 \mu \mathrm{m})=1.708$. Параметры анизометрии $\varepsilon_{y}=2.83$ и $\varepsilon_{z}=1(b=1.981 \mu \mathrm{m}$, $c=0.7 \mu \mathrm{m})$.

Из рис. 4,5 видно, что $I_{\mathrm{np}}^{\mathrm{norm}}\left(\theta_{s}, \varphi_{s}\right)$ и $P_{\text {inc }}\left(\theta_{s}, \varphi_{s}\right)$ являются периодическими функциями азимутального угла рассеяния $\varphi_{s}$. Поляризующая способность пленки $P_{\text {inc }}\left(\theta_{s}, \varphi_{s}\right)$ не зависит от полярного угла рассеяния $\theta_{s}$. Такой характер зависимости $P_{\text {inc }}\left(\theta_{s}\right)=$ const определяется условием $n_{p}=n_{\perp}$ и выбранным размером капель $a$. Свет, поляризованный ортогонально оси растяжения, не рассеивается, а поляризованный вдоль оси растяжения испытывает слабое рассеяние. При значениях $\varphi_{s}=n \pi / 4$ $(n=0,1,2, \ldots, 8)$ поляризующая способность достигает пределеных значений $P_{\text {inc }}= \pm 1$ для любых значений полярного угла рассеяния $\theta_{s}$ из рассмотренного диапазона: $-8<\theta_{s}<8^{\circ} . P_{\text {inc }}\left(\theta_{s}\right)=+1$ при $\varphi_{s}=0^{\circ}, 90^{\circ}, 180^{\circ}$, $270^{\circ}, 360^{\circ} . P_{\text {inc }}\left(\theta_{s}\right)=-1$ при $\varphi_{s}=45^{\circ}, 135^{\circ}, 225^{\circ}, 315^{\circ}$.

Предельные значения $P_{\text {inc }}\left(\theta_{s}\right)= \pm 1$ могут быть peaлизованы в достаточно широком интервале длин волн, включающем видимый и ближний инфракрасный диапазоны спектра.

На рис. 6 представлены зависимости пропускания $T_{\|, \perp}^{\mathrm{inc}}, T_{\|, \perp}, T_{\mathrm{np}}^{\mathrm{inc}}, T_{\mathrm{np}}$ и поляризации $P_{f o v}^{\text {inc }}, P_{\text {fov }}$ как функции угла поля зрения $\theta_{\text {fov }}$.

Результаты получены для растянутой пленки, содержащей монодисперсные ориентированные ЖК капли с монодоменной внутренней структурой. Параметры пленки указаны в легендах. Они определяют условия, при которых прямопрошедший свет полностью поляризован

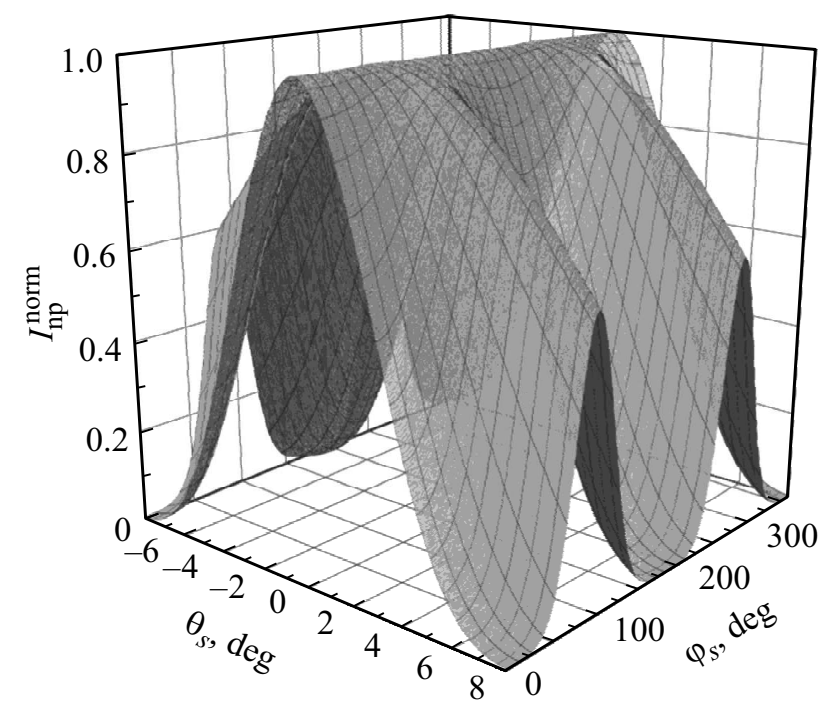

Рис. 4. Нормированная интенсивность рассеянного света $I_{\mathrm{tr}}^{\text {norm }}\left(\theta_{s}, \varphi_{s}\right)$. Монодисперсные ориентированные капли $\left(S_{2 f}=1\right)$ с монодоменной структурой. Коэффициент растяжения $p=2$. ЖК 5СВ. $a=0.7 \mu \mathrm{m}, \varepsilon_{y}=2.83, \varepsilon_{z}=1.0$ $A=B=0.5$.

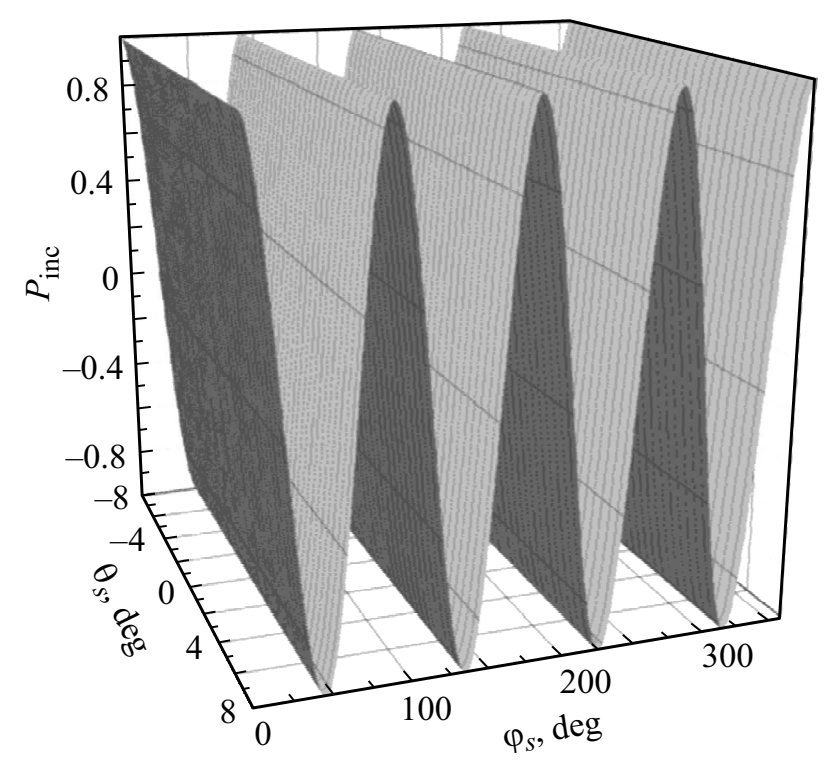

Рис. 5. Поляризующая способность $P_{\text {inc }}\left(\theta_{s}, \varphi_{s}\right)$ КПЖК пленки. Монодисперсные ориентированные капли $\left(S_{2 f}=1\right)$ с монодоменной структурой. Коэффициент растяжения $p=2$. ЖК 5СВ. $a=0.7 \mu \mathrm{m}, \varepsilon_{y}=2.83, \varepsilon_{z}=1.0 . A=B=0.5$.

$\left(P_{f o v}=1\right.$ при $\left.\theta_{f o v}=0\right)$, а коэффициент когерентного пропускания пленки $T_{c}^{\mathrm{np}}=0.5$.

Из представленных данных видно, что при угле приема $\theta_{\text {fov }}=60^{\circ}$ регистрируется практически весь диффузно рассеянный свет: полный коэффициент пропускания $T_{\mathrm{np}}\left(\theta_{\text {fov }}=60^{\circ}\right) \approx 1$. Коэффициент некогерентного пропускания для неполяризованного падающего света $T_{\mathrm{np}}^{\text {inc }} \approx 0.5$ (рис. $\left.6, c\right)$. Прямопрошедший свет является частично поляризованным со степенью поляризации 

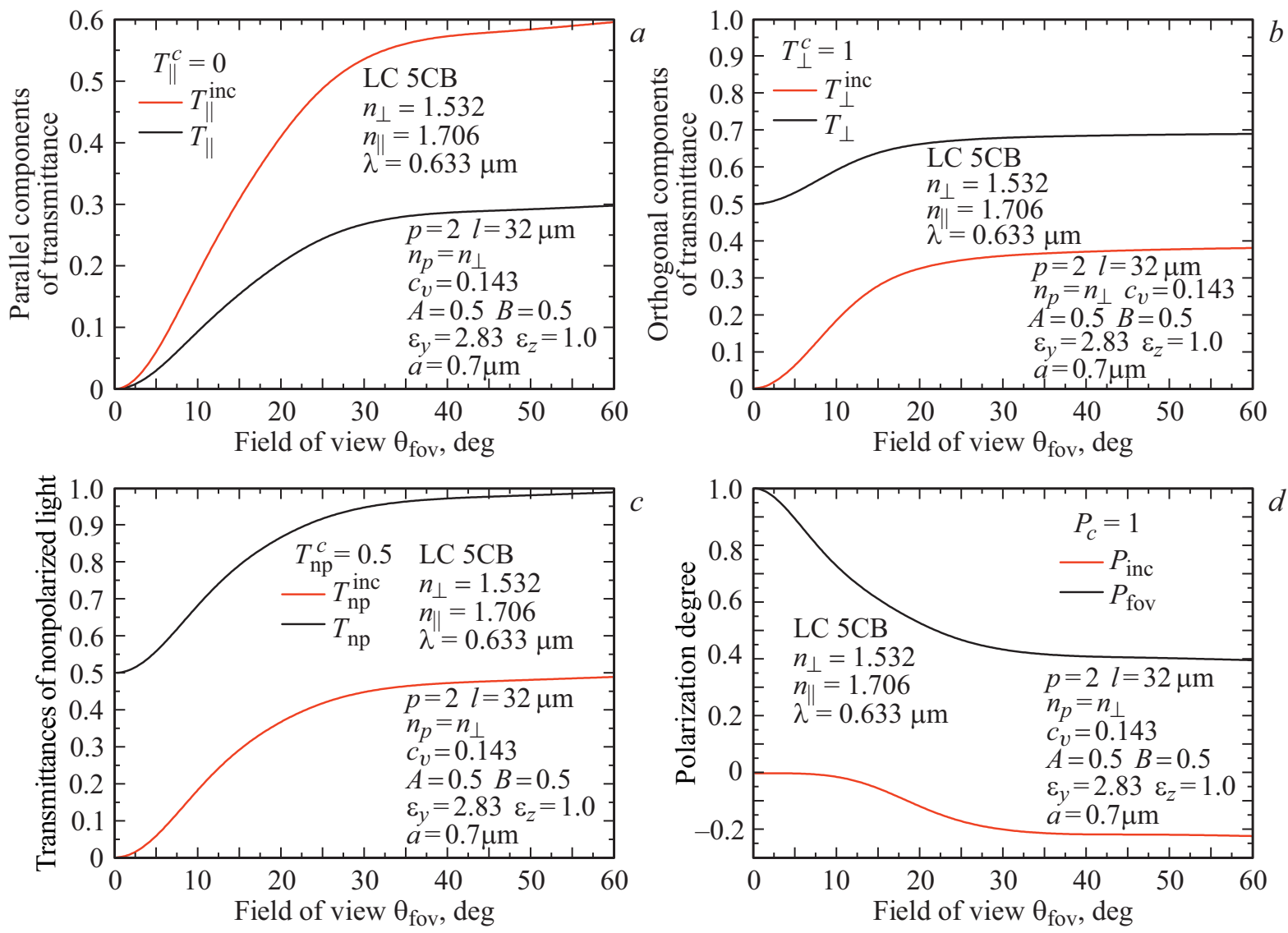

Рис. 6. Зависимости коэффициентов пропускания для параллельной $(a)$ и ортогональной $(b)$ компонент рассеянного света, коэффициентов пропускания для неполяризованного падающего света $(c)$ и степени поляризации света $(d)$ от угла поля зрения $\theta_{\text {fov }}$. Монодисперсные капли ЖК $(\mathrm{LC})$ с монодоменной структурой. Коэффициент растяжения пленки $p=2 . n_{p}=n_{\perp}=1.532$. $\lambda=0.633 \mu \mathrm{m}$.

$P_{f o v} \approx 0.4$ (рис. 6,d). Степень поляризации диффузно рассеянного света имеет отрицательное значение, приблизительно равное -0.22 , что соответствует более сильному рассеянию параллельной компоненты интенсивности диффузно рассеянного света по сравнению с ортогональной.

\section{Заключение}

Метод, изложенный в работе, позволяет рассчитывать оптимальные параметры поляроидов для конкретных практических условий их применения, когда необходимо учитывать не только когерентную часть прошедшего излучения, но и рассеянную некогерентную.

Приведены результаты численного анализа спектральных зависимостей коэффициента когерентного пропускания и поляризующей способности вытянутой непоглощающей КПЖК пленки с монодоменной структурой капель, формирующейся при ионно-сурфактантной модификации $[15,25]$ межфазного поверхностного сцепления для пленок на основе нематиков 5СВ, Е7, Е44. Результаты могут быть применены при создании поляризаторов, работающих в светорассеивающей непоглощающей моде. Они обладают высокими светостойкостью, механической прочностью, пропусканием и поляризующей способностью.

Использование КПЖК пленок, основанных на рассеянии излучения, позволяет существенно увеличить мощность поляризуемого падающего светового потока по сравнению с обычными дихроичными пленочными поляриодами, основанными на поглощении падающего излучения. Кроме того, оптический отклик КПЖК пленок можно изменять воздействием электрического поля и тем самым обеспечивать электроуправляемую модуляцию степени поляризации и пропускания света.

\section{Финансирование работы}

Работа выполнена в рамках соглашения о межакадемическом сотрудничестве между НАН Беларуси и СО РАН. Она поддержана Белорусским республиканским фондом фундаментальных исследований (проект № Ф18РА-003). М.Н. Крахалев благодарит Российский фонд фундаментальных исследований, Правительство Красноярского края и Красноярский краевой фонд науки (грант № 18-42-243006). 


\section{Конфликт интересов}

Авторы заявляют, что у них нет конфликта интересов.

\section{Список литературы}

[1] Zyryanov V.Ya., Smorgon S.L., Shabanov V.F. // Mol. Engin. 1992. N 1. P. 305.

[2] Зырянов В.Я., Шабанов В.Ф., Сморгон С.Л. // ПТЭ. 1992. № 1. C. 235.

[3] Сморгон С.Л., Пресняков В.В., Зырянов В.Я., Шабанов В.Ф. // ПТЭ. 1997. № 1. С. 164.

[4] Агабеков В.Е., Потапов А.Л., Шахаб С.Н., Иванова Н.А. // Полимерные материалы и технологии. 2015. Т. 1. № 2. C. 6 .

[5] Зырянов В.Я., Сморгон С.Л., Шабанов В.Ф. // Письма в ЖЭТФ. 1993. Т. 57. В. 1. С. 17.

[6] De Filpo G., Mashin A.I., Beneduci A., Chidichimo G. // Liquid Crystals. 2017. V. 44(9). P. 1477.

[7] Aphonin O.A., Panina Yu.V., Pravdin A.B., Yakovlev D.A. // Liquid Crystals. 1993. V. 15(3). P. 395.

[8] Bloisi F., Ruocchio C., Terrecuso P., Vicari L. // Liquid Crystals. 1996. V. 20(3). P. 377.

[9] Amimori I., Priezjev N.V., Pelcovits R.A., Crawford G.P. // J. Appl. Phys. 2003. V. 93(6). P. 3248.

[10] Klosowicz S.J., Aleksander M. // Opto-Electronics Rev. 2004. V. 2(3). P. 305.

[11] Зырянов В.Я., Крахалев М.Н., Прищепа О.О., Шабанов А.В. // Письма в ЖЭТФ. 2007. Т. 86. В. 6. С. 440.

[12] Zyryanov V.Ya., Krakhalev M.N., Prishchepa O.O. // Mol. Cryst. Liq. Cryst. 2008. V. 489. P. 273.

[13] Зырянов В.Я., Крахалев М.Н., Прищепа О.О., Шабанов А.В. // Письма в ЖЭТФ. 2008. Т. 88. В. 9. С. 688.

[14] Krakhalev M.N., Prishchepa O.O., Zyryanov V.Ya. // Mol. Cryst. Liq. Cryst. 2009. V. 512. P. 152.

[15] Эгамов М.Х., Герасимов В.П., Крахалев М.Н., Прищепа О.О., Лойко В.А., Зырянов В.Я. // Опт. журн. 2014. Т. 81. № 7. C. 67.

[16] Прищепа О.О., Эгамов М.Х., Герасимов В.П., Крахалев М.Н., Лойко В.А. // Известия вузов. Физика. 2013. Т. 56. № 2/2. C. 258.

[17] Loiko V.A., Konkolovich A.V., Zyryanov V.Ya., Miskevich A.A. // Opt. Spectrosc. 2017. V. 122(6). P. 984.

[18] Исимару A. Распространение и рассеяние волн в случайно-неоднородных средах. Т. 2. М.: Мир, 1986. 317 с.

[19] Хюлст Г. Рассеяние света малыми частицами. М.: Изд. ин. лит., 1961. 536 с.

[20] Борен Д., Хафмен К. Поглощение и рассеяния света малыми частицами. М.: Мир, 1986. 660 с.

[21] Лойко В.А., Зырянов В.Я., Конколович А.В., Мискевич А.A. // Опт. и спектр. 2016. Т. 120. № 1. С. 158.

[22] Aphonin O.A., Panina Yu.V., Pravdin A.V., Yakovlev D.A. // Liquid Crystals. 1993. V. 15. N 3. P. 395.

[23] Loiko V.A, Konkolovich A.V. // Mol. Cryst. Liq. Cryst. 1998. V. 320. P. 337.

[24] Li J., Wen C.-H., Gauza S., et al. // IEEE. 2005. V. 1. N 1. P. 51.

[25] Прищепа О.О., Бурина А.В., Крахалев М.Н., Лойко В.А., Зырянов В.Я. // Известия РАН. Серия физическая. 2017. Т. 81. № 5. C. 656-659. 